\title{
Physical mechanisms governing pattern fidelity in microscale offset printing
}

\author{
Anton A. Darhuber and Sandra M. Troian ${ }^{\mathrm{a})}$ \\ Microfluid Dynamics Laboratory, Department of Chemical Engineering, Princeton University, \\ Princeton, New Jersey 08544
}

Sigurd Wagner

Department of Electrical Engineering, Princeton University, Princeton, New Jersey 08544

(Received 7 March 2001; accepted for publication 4 June 2001)

\begin{abstract}
We have studied the offset printing of liquid polymers curable by exposure to ultraviolet light onto flat and unpatterned silicon and glass substrates. The interplay of capillary, viscous, and adhesion forces dominates the dynamics of ink transfer at small feature sizes and low capillary number. For smooth and nonporous substrates, pattern fidelity can be compromised because the ink contact lines are free to migrate across the substrate during plate separation. Using a combination of experiments and equilibrium simulations, we have identified the physical mechanisms controlling ink transfer and pattern fidelity. In considering the resolution limit of this technique, it appears that the dynamics of ink flow and redistribution during transfer do not explicitly depend on the absolute feature size, but only on the aspect ratio of film thickness to feature size. Direct printing holds promise as a high-throughput fabrication method for large area electronics. (C) 2001 American Institute of Physics. [DOI: 10.1063/1.1389080]
\end{abstract}

\section{INTRODUCTION}

Ink transfer by contact printing is the most common technique for graphical and typographical reproduction especially for high-volume printing of newspapers and books. Inherent advantages of this technique for image transfer are rapid throughput and simultaneous parallel processing of large areas. The target substrate is usually flexible and processed from reel to reel. Commercial printing presses achieve a resolution, as defined by the minimal linewidth of the printed image, on the order of $40 \mu \mathrm{m}$.

By contrast, patterns for semiconductor devices are produced by photolithography, which offers a lateral resolution down to $100 \mathrm{~nm}$. The patterns are usually defined by a sequential step-and-repeat exposure of individual fields until the entire surface of a semiconductor wafer is processed. Subsequently, the exposed photoresist layer is immersed in a developer bath, then dried, and used as an image transfer layer for etching or selective material deposition. After pattern transfer the photoresist layer must be stripped off the wafer. While the achievable resolution is orders of magnitude smaller than for conventional contact printing, the technique is only viable for patterning areas on the order of square inches.

For large area electronic applications, such as light emitting displays, the required resolution is on the order of 20 $\mu \mathrm{m}$. This feature size can be achieved without photolithography by using contact or noncontact direct patterning methods. These are designed to transfer functional material such as conductors or resistors only to desired locations of a substrate, thereby eliminating any postdeposition patterning

${ }^{a)}$ Author to whom correspondence should be addressed; electronic mail: stroian@princeton.edu steps. Direct-write methods like ink-jet printing have been used to fabricate organic light emitting diodes ${ }^{1,2}$ and contacts to silicon based thin film transistors (TFTs). ${ }^{3}$ Garnier et al. ${ }^{4}$ and Bao et al. ${ }^{5,6}$ have fabricated organic TFTs by screen printing. Kumar and Whitesides ${ }^{7}$ printed alkylthiol patterns onto gold surfaces via microcontact printing. Mikami et al. ${ }^{8}$ and Leppävuori et al. ${ }^{9}$ used a hybrid gravure-offset technique to print photolithographic masks for TFT processing. Gleskova et al. have employed laser-printed toner patterns as an etch mask in the fabrication of TFTs. ${ }^{10}$ Walton et al. have printed conductive patterns on flexible substrates like polyethylene and paper with a standard offset lithographic process. $^{11}$

In this article we present experiments and numerical simulations which elucidate the physical mechanisms governing pattern fidelity in direct printing of microscale feature sizes at small capillary number. We used offset printing to transfer patterns of ultraviolet light (UV)-curable polymers onto flat and nonporous substrates like glass or oxidized silicon wafers. Conventional offset printing involves the use of a fountain solution to cover the nonimaging regions of the printing plate and an immiscible ink, which adheres to the imaging areas. A blanket roller serves as the intermediary for image transfer. In our studies, offset printing refers to the direct transfer of liquid from a smooth, rigid, chemically patterned surface onto a smooth, rigid and unpatterned substrate without the use of a blanket roller or a fountain solution.

This type of printing process comprises five major steps: fabrication of the printing plates, dispensing of the liquid ink onto the patterned substrate, ${ }^{12}$ pattern design modifications to ensure a uniform liquid coating, ${ }^{13}$ ink transfer, and pattern fixation after printing. In what follows we address each of 
these steps in an attempt to identify the key mechanisms which influence pattern fidelity.

The numerical simulations of static equilibrium ink surface profiles were performed with SURFACE EVOLVER, ${ }^{14}$ which is an energy minimization software that computes liquid equilibrium conformations given the volume, geometric boundary conditions, and surface energies associated with the liquid-air, liquid-solid, and air-solid interfaces. Gravitational forces were ignored since the dimensionless Bond number ${ }^{15}$ is exceedingly small for feature sizes in the tens of microns range.

\section{PRINTING PLATE FABRICATION}

For both gravure and letterpress printing, liquid ink is confined to the imaging regions of the printing plate by means of a surface relief. The ink either resides inside grooves for gravure or rests atop ridges for letterpress printing. In contrast, offset printing plates are essentially flat. Liquid is instead confined to the imaging portions by chemical surface patterns, for example, by hydrophilic patterns on a hydrophobic background.

The printing plates used for this study were prepared from silicon wafers and Corning 2947 glass slides. These substrates were first cleaned by immersion in a mixture of approximately $70 \%$ sulfuric acid and $30 \%$ hydrogen peroxide at a temperature of $80^{\circ} \mathrm{C}$ for $15 \mathrm{~min}$. After rinsing in de-ionized water, the sample surfaces were further cleaned with oxygen plasma $\left(\mathrm{O}_{2}\right.$ flow rate $40 \mathrm{sccm},{ }^{16}$ pressure 100 mTorr) in a Plasmatherm 790 reactive ion etcher. Subsequently, chromium and gold were deposited with an electron beam evaporator with layer thicknesses of 5 and $40 \mathrm{~nm}$, respectively. The metal layers were then patterned by optical lithography and wet chemical etching with TFA (Transene Comp, Inc.) and CR-7 (Cyantek Corp.). After stripping off the photoresist, the samples were immersed in a $1 \mathrm{mM}$ hexadecanethiol (HDT) solution in ethanol at a temperature of $30^{\circ} \mathrm{C}$ for $45 \mathrm{~min}$. HDT forms a self-assembled monolayer on gold. ${ }^{17}$ The alkane chains of the HDT molecules are oriented away from the gold surface rendering the hydrophilic gold surface hydrophobic. The contact angle of water on HDT coated regions was measured to be $108^{\circ} \pm 3^{\circ}$. The regions where the gold and chromium layers were removed to expose the underlying $\mathrm{SiO}_{2}$ surface were completely hydrophilic with a water contact angle of $0^{\circ}$.

The masks used for the patterning of the printing plate by photolithography were printed on a polymer foil with a commercial image setter, which has a minimum feature size of about $25 \mu \mathrm{m}$. The linewidth of the hydrophilic patterns on the printing plates was $60 \mu \mathrm{m}$ with an edge roughness on the order of $5 \mu \mathrm{m}$.

\section{PATTERN GEOMETRY AND INK DEPOSITION}

The hydrophilic patterns were designed in the shape of continuous or segmented half-loops as sketched in Fig. 1. The length $L$ of the horizontal segment was 120, 240, or 480 $\mu \mathrm{m}$; the spacing $s$ between the horizontal and vertical segments ranged from 0 to $50 \mu \mathrm{m}$ in steps of $10 \mu \mathrm{m}$. The

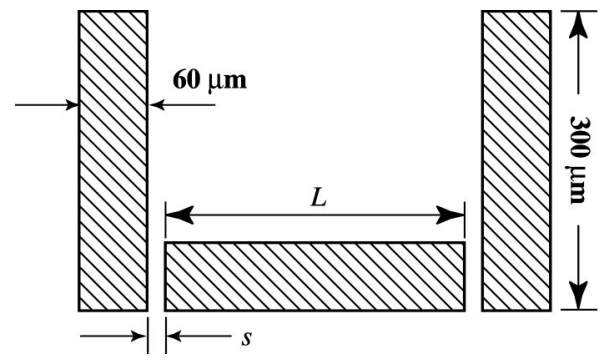

FIG. 1. Diagram of the hydrophilic surface patterns used in the experiments. The length $L$ of the horizontal segment is 120,240 , or $480 \mu \mathrm{m}$; the spacing $s$ between the horizontal and vertical segments ranges from 0 to $50 \mu \mathrm{m}$ in steps of $10 \mu \mathrm{m}$.

segmentation was introduced to avoid excess entrainment of liquid during ink deposition ${ }^{12}$ and to examine intentional pattern coalescence during printing. ${ }^{13}$

The liquids used as inks for this study are Norland optical adhesives (NOAs) No. 61 and No. 63, which are prepolymers of polyurethane. ${ }^{18}$ The viscosity $\eta$ of these liquids at $22{ }^{\circ} \mathrm{C}$ is 400 and $2000 \mathrm{mPa}$ s, respectively. Both have a surface tension $\gamma=40 \mathrm{mN} / \mathrm{m}$ and a density $\rho=1231 \mathrm{~kg} / \mathrm{m}^{3} .{ }^{19}$ The contact angles of these liquids were measured to be approximately $50^{\circ}$ on the hydrophobic HDT monolayer and $0^{\circ}$ on the hydrophilic $\mathrm{SiO}_{2}$ regions.

We selectively deposited a thin layer of these liquids onto the hydrophilic patterns by a capillary meniscus coating technique. This method is similar to dip coating ${ }^{12}$ but only requires a small ink reservoir volume. In order to speed up the deposition process, the viscosity of NOA63 was reduced by dilution with acetone, which evaporated after deposition.

\section{UNIFORMITY OF INK FILM THICKNESS}

\section{A. Time scale of capillary relaxation}

The deposition process selectively distributes a liquid volume onto the hydrophilic areas of the printing plate such that the initial surface profile does not correspond to its equilibrium shape, which would be a surface of constant mean curvature. Before the ink is transferred onto the target substrate, local differences in capillary pressure induce flow and redistribute liquid on the hydrophilic patterns. The characteristic time scale of this relaxation process depends critically on the feature size and the coating thickness, as well as the liquid surface tension and viscosity.

Orchard ${ }^{20}$ developed a theoretical model for the leveling of brush marks in thin Newtonian paint films. The surface profile was expanded into a Fourier series, whose coefficients were shown to decay exponentially in time. Under the assumption that the lateral wavelength $\lambda$ of small amplitude surface corrugations was much larger than the average film thickness $\langle h\rangle$, he derived the characteristic decay time of disturbances according to

$$
t_{\text {level }}(\lambda)=\frac{3}{16 \pi^{4}} \frac{\eta}{\gamma} \frac{\lambda^{4}}{\langle h\rangle^{3}} .
$$

The surface leveling time $t_{\text {level }}$ is linearly proportional to the viscosity $\eta$ and inversely proportional to the surface tension $\gamma$. However, there is a strongly nonlinear dependence on $\lambda$ 

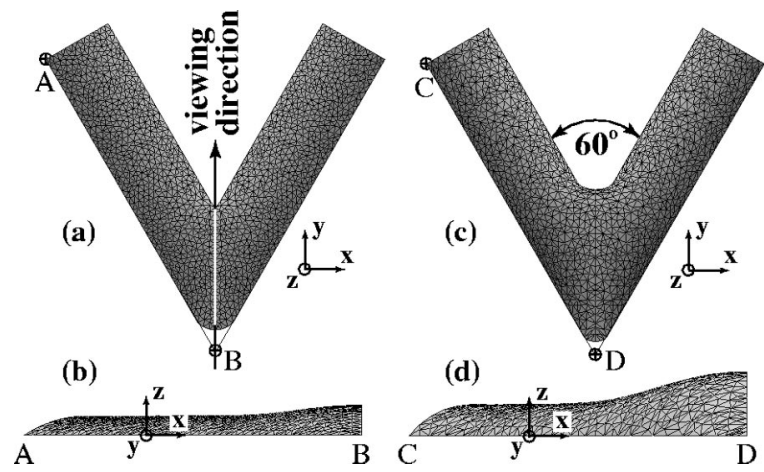

FIG. 2. Simulations of ink surface profiles at the junction of two straight line segments meeting at an angle $\varphi=60^{\circ}$. The volume fill-factor $f$ in (a),(b) is 0.1 and 0.2 in (c),(d). (a),(c) are top views; (b),(d) are side views in the viewing direction as indicated by the arrow in (a). Only the left halves of the surface profiles are shown in the side views. Corresponding points A-D are labeled in both top and side view images and the coordinate systems indicate the viewing angles.

and $\langle h\rangle$. For a value of $\lambda=120 \mu \mathrm{m}$ (twice the linewidth), $\langle h\rangle=6 \mu \mathrm{m}$ and viscosities ranging from 1 to $2000 \mathrm{mPa}$ s, the characteristic decay times range from $5 \times 10^{-5}$ to 1 $\times 10^{-1} \mathrm{~s}$. Although Orchard's model does not specifically consider liquid elements on a patterned surface, the model demonstrates that the equilibration time of a surface profile can be quite short for small feature sizes. Since in our case the time between ink deposition and transfer exceeded several minutes, the ink volume distribution corresponded to its equilibrium profile.

\section{B. Influence of the pattern geometry}

A necessary condition for pattern fidelity is the uniform distribution of ink on the hydrophilic patterns of the printing plate. The minimum separation between printing plate and target substrate that is required to transfer all parts of a pattern is determined by the minimum film thickness. When this limiting thickness is significantly smaller than the maximum film height anywhere on the plate, the thickest portions are very likely squeezed out beyond their designated boundaries causing image distortion. In the following we study which pattern shapes give rise to uneven surface profiles and how to minimize or eliminate this effect.

A common geometric pattern for interconnects in microelectronic circuits is the junction of two straight lines meeting at an angle $\varphi$. Figure 2 shows numerical SURFACE EVOLVER $^{14}$ simulations of the equilibrium surface profile of liquid deposited on two straight lines that meet at an angle $\varphi=60^{\circ}$. The contact angle $\theta$ of the liquid on the solid surfaces was chosen to be $15^{\circ}$ on the hydrophilic stripes and $100^{\circ}$ on the surrounding hydrophobic areas. To quantify the ratio of the average liquid height $\langle h\rangle$ to the stripe width $w$, we introduce the ink fill-factor $f=V /(A w)$, where $V$ denotes the deposited ink volume and $A$ the hydrophilic area. The fill-factor was $f=0.1$ in Figs. 2(a) and 2(b) and $f=0.2$ in Figs. 2(c) and 2(d). Top views are shown in Figs. 2(a) and 2(c) and side views along the viewing direction in Figs. 2(b) and 2(d).

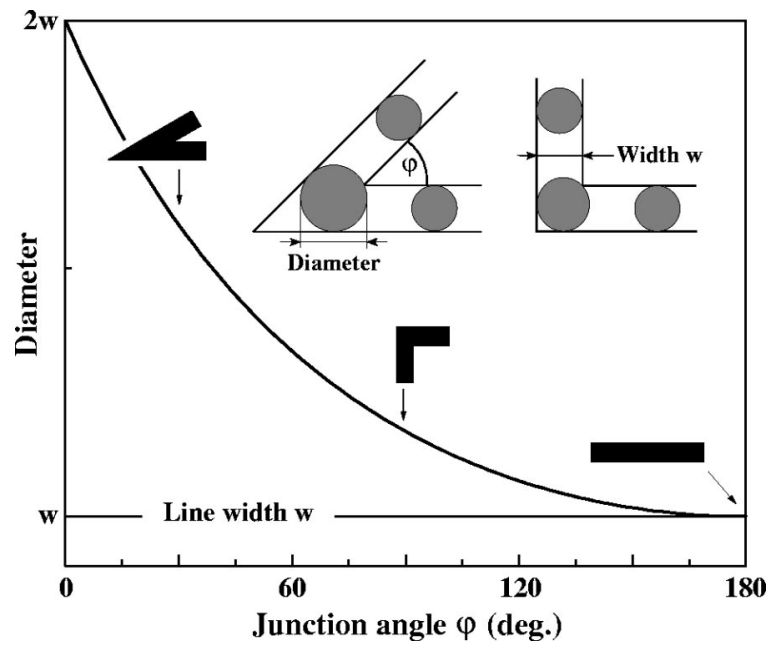

FIG. 3. The diameter of the largest circle that can be inscribed in the corner region of a junction of two lines as a function of the junction angle $\varphi$.

The side views clearly show a local maximum of the film thickness in the corner region, which becomes more pronounced as the fill-factor increases. Since the effective linewidth in the corner increases by a factor $2 /[1$ $+\sin (\varphi / 2)]$, it is energetically favorable to accumulate more liquid in this region. This concept is illustrated in Fig. 3, where the diameter of the largest circle that fits into the hydrophilic corner region is plotted as a function of the junction angle $\varphi$. For $\varphi=90^{\circ}$ the diameter is about $20 \%$ larger than the channel width. The inscribed diameter progressively increases for smaller junction angles and the relative change in the height of the ink profile scales as the square of the relative change in width of the hydrophilic pattern. Figure 2(b) shows that for relatively large values of the fill-factor $f=0.2$, the liquid is forced to spread into the hydrophobic wedge, which in view of Fig. 3 further amplifies the bulging effect.

Acute junctions connecting hydrophilic stripes or a small radius of curvature of hydrophilic/hydrophobic boundaries must therefore be avoided to maintain pattern fidelity. Figures 2 and 3 together indicate that the ideal junction geometry requires the radius of an inscribed circle to be constant. Figure 4 shows simulations of the ink surface profile on a rounded junction for a fill-factor of $f=0.2$. The surface does

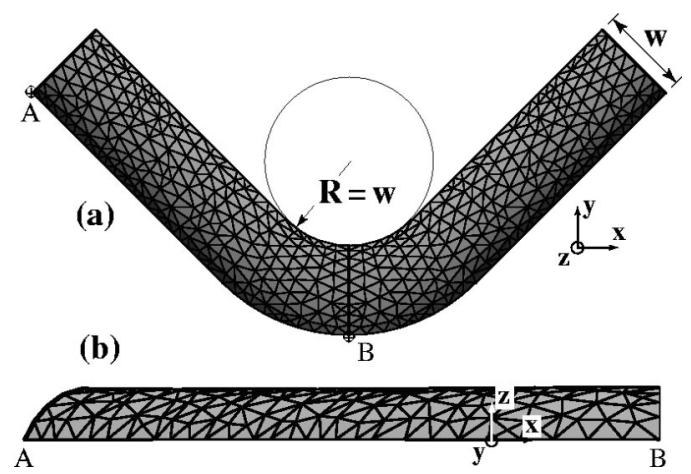

FIG. 4. Simulation of an ink surface profile at the rounded junction of two perpendicular lines. The fill-factor $f$ is 0.2 : (a) top view, (b) side view in the same orientation as Fig. 2(b). 


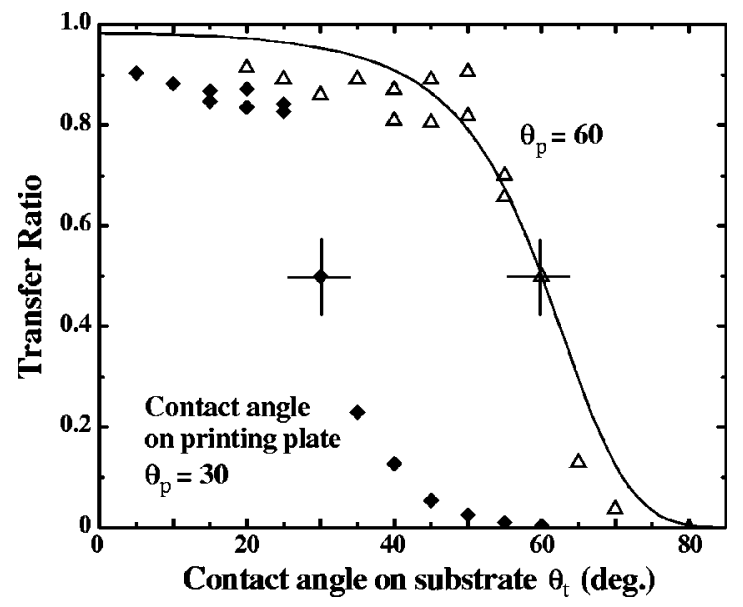

FIG. 5. Simulation of the ink transfer ratio $\mathcal{R}$ as a function of $\theta_{t}$ for two values of $\theta_{p}=30^{\circ}$ (diamonds) and $60^{\circ}$ (triangles). The solid curve represents the function $K_{T, 1}$ for $\theta_{p}=60^{\circ}$ according to Ref. 22 .

not exhibit a corner bulge but maintains a flat profile. The radius of curvature of the inner hydrophilic/hydrophobic boundary was set equal to the channel width. For large fillfactors, however, bulges can still form in corner regions. ${ }^{21}$

\section{INK TRANSFER PROCESS}

\section{A. The influence of surface energies on quasistatic ink transfer}

Chadov and Yakhnin ${ }^{22,23}$ investigated the transfer and volume partition of liquid droplets in the $\mathrm{mm}$ size range from one unpatterned solid surface to another for varying rates of plate separation. The fastest separation rates showed that the liquid volume was equally partitioned among the two surfaces. For slow separation rates such that quasiequilibrium conditions were maintained, they found that the volume partition was completely determined by the receding contact angles on the respective surfaces. In the limit of small Bond number, the partition ratio was independent of the liquid volume used. They further developed a simple geometric model for the transfer coefficient for quasistatic printing. ${ }^{22}$

From a hydrodynamic point of view, quasistatic transfer of a liquid from one solid surface to another corresponds to flow at low Reynolds Re and very low capillary number $\mathrm{Ca}$, where $\mathrm{Ca}=\eta v_{\mathrm{ps}} / \gamma$. Here $v_{\mathrm{ps}}$ denotes the rate of plate separation. In this regime, capillary forces dominate over viscous forces implying that the volume partition is determined only by the surface energies. For surfaces which are flat and smooth, such that topological features like roughness and porosity are absent, the liquid contact angle on the printing plate $\theta_{p}$ and on the target surface $\theta_{t}$ completely determine the volume transfer ratio $\mathcal{R}=V_{\text {transferred }} / V_{\text {total }}$.

In Fig. 5 we present simulations of $\mathcal{R}\left(\theta_{t}\right)$ for liquid droplets transferred from one surface (printing plate) to another (target substrate) for two values of $\theta_{p}=30^{\circ}$ and $60^{\circ}$. Both solid surfaces were assumed to be unpatterned, i.e., chemically homogeneous. The geometrical model devised by Chadov and Yahknin ${ }^{22}$ assumes that the vertical crosssectional profile of the liquid meniscus, which forms upon detachment, resembles two circular arcs. The solid line in
Fig. 5 corresponds to the transfer coefficient function $K_{T, 1}$ given in Ref. 22 for $\theta_{p}=60^{\circ}$. The simulation data closely approximate the curve obtained from this geometric model of liquid transfer at quasistatic conditions.

The scatter in the data obtained from the simulations is mainly due to the mesh refinement and adaptation procedure used to discretize the surface profiles during the energy minimization calculations. Despite this scatter, certain general trends are evident. If $\theta_{t}=\theta_{p}$, the transfer ratio is exactly $50 \%$ as dictated by symmetry. For $\theta_{t}>\theta_{p}$, it is energetically favorable for the ink to remain on the printing plate in which case $\mathcal{R}$ sharply decreases to zero. Conversely, $\mathcal{R}$ rapidly approaches 1 for $\theta_{t}<\theta_{p}$. The transfer ratio $\mathcal{R}$ can never exactly equal unity since for contact angles $\theta_{t}$ and $\theta_{p}$ smaller than $90^{\circ}$, the snap-off point of the liquid meniscus is always located inbetween the surfaces. $\mathcal{R}=1$ only occurs for $\theta_{p}$ $\geqslant 90^{\circ}$, which would correspond to a hydrophobic printing plate. From a practical point of view, it is unfavorable to have equal contact angles $\theta_{p}=\theta_{t}>0^{\circ}$ on both plates, since small deviations will have a large influence on $\mathcal{R}$. The preferable stable operating range is therefore $\theta_{t}<\theta_{p}$.

\section{B. Control of plate separation}

Since the surfaces used in this study are flat, smooth, and nonporous, ink patterns are squeezed beyond the designated boundaries when the separation between the printing plate and the target substrate becomes too small. The simplest way to maintain a minimum plate separation is to introduce rigid spacer elements on the printing plate as shown in Fig. 6(a). The spacers should preferably be hydrophobic to avoid liquid entrainment during ink deposition and subsequent undesirable transfer. The thickness of the spacers must match the plate separation required for faithful reproduction of the ink patterns.

The optimal separation depends not only on the volume of ink confined to the hydrophilic patterns and their feature size but also on the pattern geometry. In Fig. 6(a), we consider two limiting cases of geometric aspect ratios: a circular pad and a stripe whose length far exceeds its width. The equilibrium profile of liquid on a circular pad is a spherical cap. As the printing plate approaches the target surface, ink will be redistributed isotropically, i.e., in the twodimensional plane parallel to the plates. The equilibrium profile of liquid on a long hydrophilic stripe is a segment of a circular cylinder. In this case, the printing process redistributes the ink only in the direction normal to the long axis of the stripe. Because the liquid only spreads in one direction, the optimal spacer thickness will be smaller for circular pads than for long stripes even for the same apparent contact angle $\theta_{a}$, provided the linewidth equals the circle diameter.

Shown in Fig. 6(b) are simulation results of the optimal spacer thickness as a function of the apparent contact angle $\theta_{a}$ of the liquid confined to a hydrophilic area. The spacer height is normalized by the relevant feature width (i.e., the width of the long stripes or the diameter of the circular pads). The angle $\theta_{a}$ is a measure of the ink volume deposited. The solid curves are obtained from simulations for circular pads. The dotted lines represent analytic results which are easily 

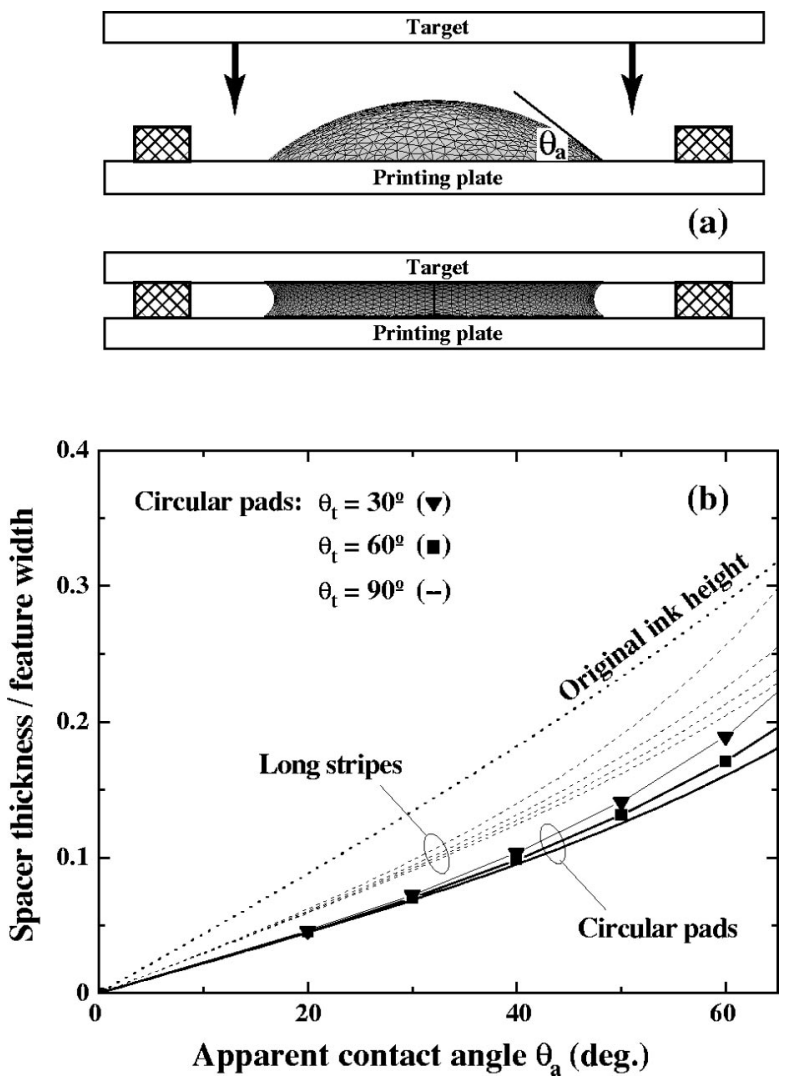

FIG. 6. (a) Spacer elements (cross hatched) enforce the proper separation of plates required for faithful pattern reproduction. (b) Optimal spacer thickness for long stripes (dashed lines) and circular pads (solid lines) as a function of the apparent contact angle $\theta_{a}$. The dotted line is the apex height of the ink features prior to contact of the plates. The two sets of curves correspond to contact angles on the target substrate of $\theta_{t}=0^{\circ}$ (bottom), $30^{\circ}, 60^{\circ}$, and $90^{\circ}$ (top).

derived for long stripes since the menisci form arcs of a circle. The technologically relevant range in contact angles is $0<\theta_{a} \lesssim 15^{\circ}$. Within this interval, the optimal spacer thickness is essentially independent of the contact angle on the target substrate. However, there is a difference of about $20 \%$ in the optimal plate separation between lines and circular dots. Therefore, if lines and dots are to be printed simultaneously, the pattern design must compensate for this difference.

Figure 7(a) is a photograph of an ink meniscus in transfer from a hydrophilic rectangular pad to an unpatterned glass slide. The target surface is transparent and contains no spacer elements. The plate separation was so small that the ink was squeezed beyond the boundaries of the hydrophilic region. The ink was primarily displaced in a direction perpendicular to the stripe. This preferential spreading continues until the liquid-solid contact lines adopts a circular shape. From that point on, progressive squeezing displaces the ink isotropically. The faint outer perimeter in Fig. 7(a) is the liquid-solid contact line on the target substrate. The liquid extended beyond the contact line on the printing plate because $\theta_{t} \approx 0$, whereas the contact angle on the hydrophobic regions was approximately $50^{\circ}$. Moreover, the target plate was slightly retracted from the substrate before the image was recorded. Figure 7(b) represents a top view of the cor-

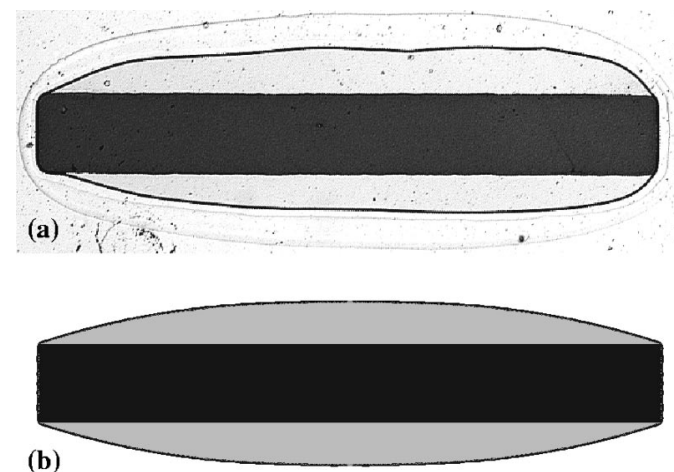

FIG. 7. Ink contact line for printing without spacers. (a) Optical micrograph. Because the separation of stamp and target substrate was too small, the ink contact line was squeezed beyond the boundaries of the hydrophilic rectangle. (b) Corresponding numerical simulation. The width of the rectangle is $500 \mu \mathrm{m}$.

responding SURFACE EVOLVER simulation for the common perimeter for the case of no retraction.

\section{Contact line motion upon plate separation}

Ink transfer is accompanied by liquid flow both perpendicular and parallel to the printing plate and target substrate. The roughness and porosity of a substratelike paper can prevent ink contact line migration during plate separation. In our case the target surface is smooth and flat. Thus contact lines can move freely, especially for partially wetting inks, which can effectively diminish pattern fidelity. Simulations of the deformation of a liquid meniscus during a quasistatic separation of the plates are shown in Fig. 8. The liquid was confined to a hydrophilic rectangle on the printing plate and the contact angles were assumed to be $30^{\circ}$ on both surfaces. Each frame in Fig. 8 represents an ink meniscus in equilibrium, i.e., a surface of constant mean curvature. The initially rectangular ink meniscus is drawn into the center of the hydrophilic line segment as the plates are moved apart and resembles more and more an axisymmetric surface. The

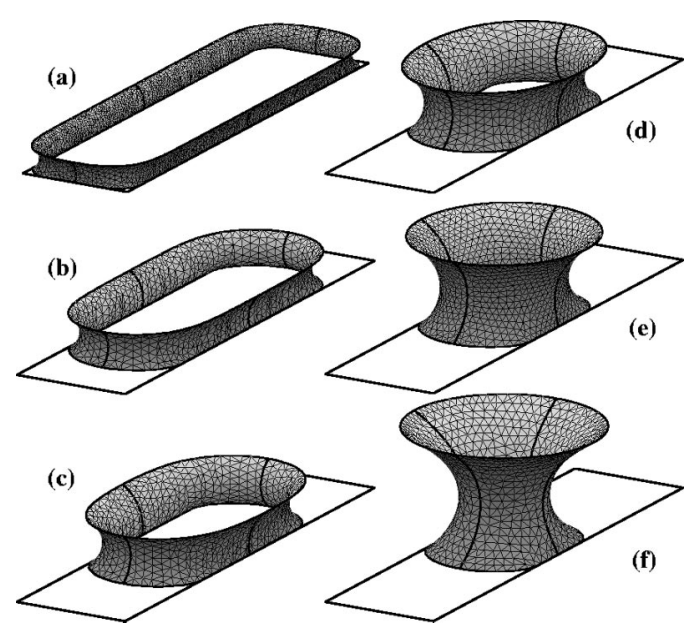

FIG. 8. Image sequence of quasistatic simulations for an ink meniscus deforming between partially wetting plates for increasing plate separation $d$. Only the bottom plate surface is shown for better visualization. As $d$ increases, the contact lines move inwards and the contact line shape on the target substrate becomes circular. 

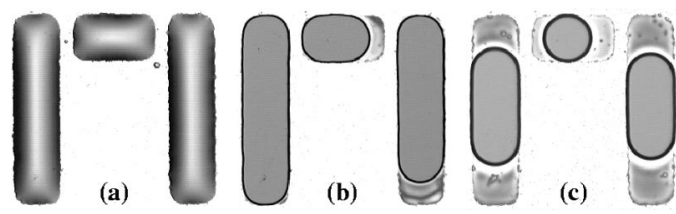

FIG. 9. Photographs of ink patterns and menisci. (a) Liquid patterns on a printing plate before contact with the target substrate. (b) Ink menisci between the printing plate and a transparent target for a plate separation close to the optimal value. (c) Meniscus narrowing for larger plate separation. The ink recedes towards the centers of the stripes as illustrated in Fig. 8.

printed pattern is therefore severely distorted when the contact line is free to recede on the target surface. Figure 9 demonstrates this distortion during plate separation. As the printing and target plate are pulled further apart, liquid migrates toward the center of each of the hydrophilic stripes.

Pattern fidelity can be improved by tuning the dynamical parameters governing the printing process. Generally, a contact line will recede from a surface only when the dynamic (receding) contact angle falls below a minimum value $\theta_{\min }{ }^{24}$ Moreover, the contact line speed decreases with decreasing contact angle since viscous dissipation increases. This recession of the contact line can therefore be reduced or suppressed by using an ink which completely wets the target surface because in this case $\theta_{\min }=0$.

A second dynamical parameter which can improve pattern fidelity is the rate of plate separation. The simulations shown in Fig. 8 demonstrate that when the plate separation is performed very slowly, the contact lines recede inward and the ink redistributes toward the center of the rectangle. If the plate separation velocity is much higher than the intrinsic contact line speed, this volume redistribution can be reduced. The contact line speed is determined by the liquid viscosity, surface tension, and $\theta_{\min } \cdot{ }^{25}$ Rapid plate separation induces a narrowing of the menisci whose speed can exceed the speed of the contact line. In this case, the ink transfer ratio is $50 \%$ for printing from unpatterned surfaces irrespective of the chemical composition of the ink or the surface. ${ }^{23}$ Sharma has calculated the shape of the liquid meniscus between two circular plates separated at constant speed. ${ }^{26}$ Under the assumption of stationary contact lines, the surface profile prior to pinch-off resembles a double cone extending to the edges of the plates. In this high shear rate limit, a significant volume of liquid remains at the outer perimeter of the circular plates. This cone shape was also observed in the experiments of Yakhnin and Chadov [Fig. 4(a) in Ref. 23].

Consequently, if viscous forces also contribute to flow dynamics, some liquid will be left at the margins of the hydrophilic pattern as the meniscus narrows. For a long stripe, the ink transfer is then a process analogous to the dip coating of hydrophilic lines as described in Ref. 12. In this case, as the meniscus necking velocity increases with increasing plate separation speed $v_{\mathrm{ps}}$, more and more liquid is entrained along the entire stripe.

Figure 10 shows photographs of half-loop patterns printed onto glass slides with a parallel plate assembly on a motorized translation stage. The spacer elements for these experiments were photoresist lines (Clariant AZ-5214) lo-

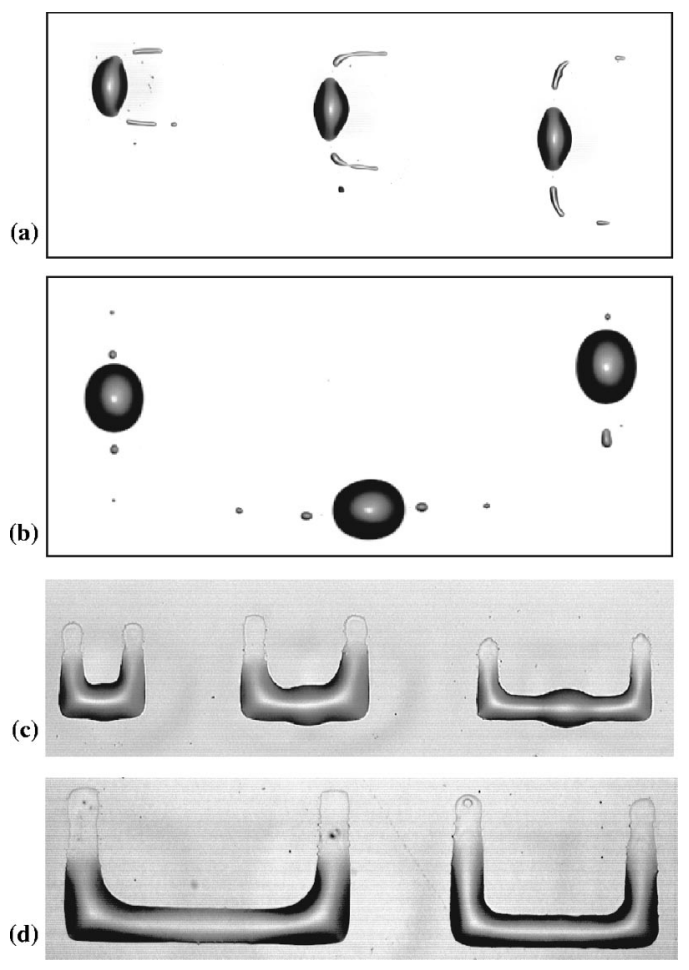

FIG. 10. Photographs of polymer patterns printed onto glass. (a),(b) The partially wetting target surface led to strong pattern distortions. The three rectangles of the segmented half-loop in (b) each produced a central ink dot with small satellite droplets. (c),(d) Completely wetting target surface. The ink patterns were precured for (c) $8 \mathrm{~s}$ and (d) $20 \mathrm{~s}$ on the printing plate after deposition and before printing.

cated at the margins of the target substrates. In Figs. 10(a) and $10(\mathrm{~b})$, the NOA61 polymer wets the target surface only partially $\left(\theta_{\min }>0\right)$. As discussed above, strong pattern distortions occur during plate separation. In these examples, most of the ink volume was redistributed onto the central rectangle of the half-loop with contact lines resembling circles rather than rectangles. As shown in Fig. 10(a), the dewetting process left behind only thin filaments where there previously were liquid rectangles. These filaments originate from capillary breakup which also created the satellite droplets in Fig. 10 (b).

The patterns depicted in Figs. 10(c) and 10(d) were printed with NOA63, the viscosity of which is five times higher than that of NOA61. In addition, the target substrate was completely wetting $\left(\theta_{t}=0\right)$ which made the transferred patterns resemble the designed half-loops much more closely. The ink viscosity was further increased by precuring the polymer patterns after deposition on the printing plate but before printing. The precure time was $8 \mathrm{~s}$ for Fig. 10(c) and $20 \mathrm{~s}$ for Fig. 10(d). A comparison of Figs. 10(c) and 10(d) shows that a higher viscosity is advantageous for increasing the fidelity between printed and designed patterns as outlined above. Alternatively, lower fill factors will have the same effect; however, both approaches increase the force required for plate separation.

As shown in Fig. 10(d), we have successfully printed U-shaped patterns with feature sizes of $60 \mu \mathrm{m}$ onto completely wetting glass and oxidized silicon samples. This linewidth does not represent the resolution limit of this tech- 
nique. For conventional offset printing, the printed ink film thickness is on the order of $1 \mu \mathrm{m}$ for typical feature sizes of $100 \mu \mathrm{m}$ or larger. The fill factor $f$ is typically 0.01 , whereas in our process, $f$ ranges between 0.01 and 0.1 . Since inertial and gravitational forces are negligible in the limit of small aspect ratios and small feature sizes, only capillary and viscous forces affect the printing dynamics. According to the Buckingham $\Pi$-theorem ${ }^{27}$ this leads to a functional relation between the capillary number and the fill factor. The characteristic velocity for ink redistribution is therefore $v$ $=\gamma G(f) / \eta$ independent of feature size. The dimensionless function $G$ depends on the specific flow geometry. For Orchard's one-dimensional model, the spreading of an axisymmetric droplet on a flat wettable surface, ${ }^{28}$ or the steady-state dip coating of long hydrophilic stripes, ${ }^{12} G(f) \sim f^{3}$. This scaling argument leads to the expectation that the velocity required for faithful pattern reproduction does not depend on the actual feature size but just on the relevant aspect ratios. However, as the linewidth is reduced, the spacer thickness must be decreased proportionally, making it more challenging to maintain a constant plate separation over large areas. For ultrathin liquid films below about $100 \mathrm{~nm}$, the scaling relation may have to be modified to account for additional disjoining or electrostatic forces. ${ }^{29}$

\section{PATTERN FIXATION}

In Sec. $\mathrm{V}$ we described the advantage of using inks which completely wet the target substrate. Unfortunately, such inks will also cause the transferred pattern to spread on the target surface after printing. This undesired spreading can be prevented and the pattern affixed by inducing a rapid increase in the ink viscosity, which is usually accomplished by solidification. Common methods include penetration and imbibition into porous substrates, oxidation, microwave or infrared radiation assisted solvent evaporation and drying, and $p \mathrm{H}$-controlled precipitation. ${ }^{30}$ Alternatively, surface induced reactions of the ink with a suitable layer on the target surface could be used to affix printed patterns even on partially wetting substrates.

The inks used for this study were UV-curable at a wavelength $\lambda \approx 350 \mathrm{~nm}$. In general, curing times depend on the film thickness, the type and concentration of photoinitiator, and the power of the light source. We used a low-power UV lamp (115 V, $0.16 \mathrm{~A}, \lambda=365 \mathrm{~nm})$ with a light intensity of approximately $1 \mathrm{~mW} / \mathrm{cm}^{2}$ at a distance of $15 \mathrm{~cm}$. The curing process initiates at the ink surface and progresses inward. ${ }^{19}$ This polymerization process efficiently pins the contact lines to the target surface, thus suppressing any spreading of printed patterns. Without curing, the ink patterns spread approximately to twice their originally printed feature size within $20 \mathrm{~min}$. For smaller patterns the relative spreading rate $1 / w_{0}(\partial w / \partial t)$ is higher, thus reducing the time available for solidification, before pattern distortions become significant.

\section{SUMMARY}

In this article, we have studied static and dynamic aspects of offset printing onto flat, smooth, and rigid substrates for feature sizes in the tens of micron range. At these small scales, gravitational and inertial forces do not contribute to the dynamics of liquid transfer. Only capillary, viscous, and adhesion forces govern ink redistribution during and after printing. Our experiments and equilibrium simulations suggest that the key parameters affecting ink transfer and pattern fidelity are the uniformity of the ink profile on the patterned printing plate, the wetting angle on the target surface, and the ink viscosity. Nonuniformities in the ink thickness can lead to incomplete transfer or undesired squeeze-out effects. Partially wetting target surfaces can induce severe pattern distortions due to contact line recession during plate separation. The experiments suggest that higher ink viscosity, lower deposited ink volume, or increased rate of plate separation will enhance viscous effects thereby minimizing unwanted lateral ink redistribution and migration during the transfer process. A scaling argument for the interplay of capillary and viscous forces indicates that pattern fidelity can be retained at much smaller length scales, since the characteristic velocity of ink redistribution depends only on the relevant aspect ratio of film thickness to feature size and not the absolute feature size.

\section{ACKNOWLEDGMENTS}

This project was funded by the Molecular Level Printing program of the Defense Advanced Research Projects Agency (DARPA) and the New Jersey Center for Science and Technology Program for Large Area Electronics. Additional funding from the National Science Foundation XYZ on a Chip program (Grant No. CTS-0088774) and a Princeton University MRSEC grant (No. DMR-9809483) is gratefully acknowledged. We also thank the Austrian Fonds zur Förderung der Wissenschaftlichen Forschung for a postdoctoral fellowship.

${ }^{1}$ T. R. Hebner, C. C. Wu, D. Marcy, M. H. Lu, and J. C. Sturm, Appl. Phys. Lett. 72, 519 (1998)

${ }^{2}$ J. Bharathan and Y. Yang, Appl. Phys. Lett. 72, 2660 (1998).

${ }^{3}$ C. M. Hong and S. Wagner, IEEE Electron Device Lett. 21, 384 (2000).

${ }^{4}$ F. Garnier, R. Hajlaoui, A. Yassar, and P. Srivastava, Science 265, 1684 (1994).

${ }^{5}$ Z. Bao, Y. Feng, A. Dodabalapur, V. R. Raju, and A. J. Lovinger, Chem. Mater. 9, 1299 (1997).

${ }^{6}$ Z. Bao, J. A. Rogers, and H. E. Katz, J. Mater. Chem. 9, 1895 (1999).

${ }^{7}$ A. Kumar and G. M. Whitesides, Appl. Phys. Lett. 63, 2002 (1993).

${ }^{8}$ Y. Mikami et al., IEEE Trans. Electron Devices 41, 306 (1994).

${ }^{9}$ S. Leppävuori, J. Väänänen, M. Lahti, J. Remes, and A. Uusimäki, Sens. Actuators A 41-42, 593 (1994).

${ }^{10} \mathrm{H}$. Gleskova, R. Könenkamp, S. Wagner, and D. S. Shen, IEEE Electron Device Lett. 17, 264 (1996)

${ }^{11}$ A. J. Walton, J. T. Stevenson, L. I. Haworth, M. Fallon, P. S. A. Evans, B. J. Ramsey, and D. J. Harrison, IEICE Trans. Electron. 82-C, 576 (1999); B. J. Ramsey, P. S. A. Evans, and D. J. Harrison, J. Electron. Manuf. 7, 63 (1997).

${ }^{12}$ A. A. Darhuber, S. M. Troian, J. M. Davis, S. M. Miller, and S. Wagner, J. Appl. Phys. 88, 5119 (2000).

${ }^{13}$ A. A. Darhuber, S. M. Troian, S. M. Miller, and S. Wagner, J. Appl. Phys. 87, 7768 (2000).

${ }^{14}$ K. Brakke, Exp. Math. 1, 141 (1992).

${ }^{15}$ R. F. Probstein, Physicochemical Hydrodynamics (Butterworths, Stoneham, 1989).

${ }^{16}$ The abbreviation $\mathrm{sccm}$ denotes a standard cubic centimeter per minute. The gas flow rate $Q_{s}$ in sccm is defined as $\dot{m} / \rho_{s}$, where $\dot{m}$ is the mass flow rate and $\rho_{s}$ is the gas density at standard conditions. Standard con- 
ditions are typically defined as a pressure of $1 \mathrm{~atm}$ and a temperature of $25^{\circ} \mathrm{C}$.

${ }^{17}$ C. D. Bain, E. B. Troughton, Y.-T. Tao, J. Evall, G. M. Whitesides, and R. G. Nuzzo, J. Am. Chem. Soc. 111, 321 (1989).

${ }^{18}$ Y. Xia, M. Mrksich, E. Kim, and G. M. Whitesides, J. Am. Chem. Soc. 117, 9576 (1995).

${ }^{19}$ Norland optical adhesives technical information sheets.

${ }^{20}$ S. E. Orchard, Appl. Sci. Res. A11, 451 (1962).

${ }^{21}$ H. Gau, S. Herminghaus, P. Lenz, and R. Lipowsky, Science 283, 46 (1999).

${ }^{22}$ A. V. Chadov and E. D. Yakhnin, Kolloidn. Zh. 41, 817 (1979).

${ }^{23}$ E. D. Yakhnin and A. V. Chadov, Kolloidn. Zh. 45, 1183 (1983).
${ }^{24}$ A. W. Adamson, Physical Chemistry of Surfaces (Wiley, New York, 1993).

${ }^{25}$ F. Brochard-Wyart and P. G. de Gennes, Adv. Colloid Interface Sci. 39, 1 (1992).

${ }^{26}$ S. P. Sharma, Proceedings of the 20th Annual Holm Seminar on Electrical Contacts (Illinois Institute of Technology, Chicago, 1974), p. 89.

${ }^{27}$ R. B. Bird, W. E. Stewart, and E. N. Lightfoot, Transport Phenomena (Wiley, New York, 1960), p. 185.

${ }^{28}$ P. G. deGennes, Rev. Mod. Phys. 57, 289 (1985).

${ }^{29}$ J. Israelachvili, Intermolecular \& Surface Forces (Academic, San Diego, 1992).

${ }^{30}$ The Print and Production Manual, edited by M. Barnard (Pira, Leatherhead, 1998). 